\title{
Performance Evaluation of new Energy Aware Cluster Based Multi-hop (EACBM) Routing Protocol in Wireless Sensor Networks
}

\author{
Amanjot Singh Toor ${ }^{1}$ and A.K. Jain ${ }^{2}$ \\ ${ }^{1}$ PhD Scholar, Department of Instrumentation \& Control, Dr. B.R Ambedkar \\ National Institute of Technology, Jalandhar, Punjab, India \\ ${ }^{2}$ Professor, Department of Instrumentation \& Control, Dr. B.R Ambedkar \\ National Institute of Technology, Jalandhar, Punjab, India \\ 1er.amanjot87@gmail.com, ${ }^{2}$ jainak@nitj.ac.in
}

\begin{abstract}
Wireless Sensor Networks (WSNs) have turn out to be an integral part of our lives as they are used in every part of applications varying from disaster management to medical sciences. In such applications, network lifetime and energy efficiency have become the important requirements of WSNs. Hierarchical cluster based routing is mostly used to take care of these issues where sensor nodes are organized to form clusters and a particular sensor node is selected inside each cluster for inter and intra-cluster communication. In this paper, new hierarchical cluster based energy efficient routing protocol i.e. Energy Aware Cluster Based Multi-hop (EACBM) is proposed which uses new probabilistic equation along with the concept of clustering and multi-hoping. Here multi-hoping is used for inter-cluster communication for transferring information from Cluster Head (CH) and Base Station (BS) via number of hops if $\mathrm{CH}$ lies far away from $B S$. This results in reducing the number of transmission, due to which network lifetime and energy efficiency is increased. The concept of sub-clustering is also used here to cover those sensor nodes which are deployed far away during their random distribution phase as those far way sensor nodes may gather information which may be very crucial for applications such as military application. Simulation results shows that EACBM routing protocol performs better when compared with other cluster based energy efficient routing protocols (LEACH, SEP, CEEC and LEFCA) in terms of network lifetime, packets transmitted to $B S$, number of $C H s$, stability period and number of dead sensor nodes per round.
\end{abstract}

Keywords: Wireless Sensor Networks (WSNs); Base Station (BS); Sensor nodes (SNs); Cluster Head (CH); Energy-efficient; Clustering

\section{Introduction}

Wireless Sensor Networks (WSNs) are used for observing the physical world via various modest, cheap and smart sensor nodes which are scattered in specific territory of interest and are broadly utilized as a part of various applications, for example, natural surroundings and industry observing, medicinal determination, condition checking and agriculture [1]. Their sensor nodes are self-sufficiently used to detect, handle and remotely pass on environment conditions to a Base Station. They are comprised of three subsystems; a sensing unit to fetch the information from physical condition, a processing unit to process and handle the aggregated data and a transmitting unit to transmit the data [2]. These nodes are normally controlled

Received (December 5, 2017), Review Result (February 2, 2018), Accepted (February 5, 2018) 
by the limited power supply or battery whose replacement is difficult in hostile situation [3]. Therefore, the major concern is that how to enhance the network lifetime of WSNs with these batteries operated sensor nodes so that they can observe the sensing area for longer time. Other related issues and challenges in WSNs are like energy efficiency, coverage, scalability, fault-tolerance, connectivity, robustness, security and so on [1].

Routing in WSNs is one of the energy effective methods which are used to bring down the communication energy burden on sensor nodes [4]. Most common types of routing are flat-based, location-based and cluster-based routing (also called hierarchical routing) [5].

The cluster-based routing is broadly utilized in WSNs for improving network lifetime due to its energy efficiency and load balancing feature. In cluster-based routing, all sensor nodes are well organized to form a group called cluster in which, a Cluster Head $(\mathrm{CH})$ is selected which act as the leader to all other remaining sensor nodes. The main aim of this $\mathrm{CH}$ is to collect and aggregate all the information from its own cluster member sensor nodes and forward it to the Base Station (BS) [1,6-9]. This process of aggregating the information is useful in reducing the amount of information for transmission to BS. With this energy dissipation in communic ation is reduced and accomplishes the purpose of saving energy of sensor nodes and have enhanced network lifetime [10].

Clustering in WSNs can be homogeneous or heterogeneous clustering. For having longer network lifetime in homogeneous sensor network, it can be done by increasing the number of sensor nodes in specific application. This can increase the energy of sensor network however cost is also increased. Therefore, instead of increasing the number of sensor nodes having same energy, it is more suitable and efficient to deploy few sensor nodes having high energy. The network having such kind of qualities i.e., sensor nodes with different energy levels are called as heterogeneous sensor networks [2]

Mostly, a cluster-based routing utilizes single hop routing between $\mathrm{CH}$ and BS. This single hop communication results in more energy consumption when the communication distance between $\mathrm{CH}$ and $\mathrm{BS}$ increases and turns to be less efficient method for conserving energy. For this reason multi-hop communication is proposed in cluster based routing to enhance the network lifetime by saving its transmission energy [11].

Some of the sensor nodes may not have joined any clusters during cluster formation phase or maybe they lie far away from $\mathrm{CH}$ range. It's important to cover those sensor nodes because may be those sensor nodes contains information which is of great importance in some particular type of application (like military application). The concept of Sub-clustering is used to cover those far away sensor nodes [12].

Energy saving in Cluster based routing is a challenging issue while designing a new energy efficient protocol for WSNs which provides less collision and interference in the network and helps in reducing the redundancy which results in the improvement of network lifetime [5,13].

In this paper, the proposed approach combines 3-level heterogeneous network with clustering, sub-clustering and multi-hop communication technique for improving the network lifetime of WSNs.

Rest of the paper is arranged into following sections: Related work is described in Section II. The proposed algorithm is discussed in section III. In Section IV, simulation and performance comparison of EACBM with other cluster based routing protocols are done. Lastly, Section V concludes the paper. 


\section{Related Work}

Various cluster based routing protocols and approaches have been developed by researchers to improve energy and enhancing network lifetime and are summarized below.

In 2000, the foremost accepted cluster based routing protocol called Low Energy Adaptive Clustering Hierarchy $(\mathrm{LEACH})$ was proposed by Heinzelman et al., for enhancing the network lifetime in WSNs [14]. In this homogeneous sensor nodes are used which is divided into many groups called clusters and inside each cluster there is Cluster Head $(\mathrm{CH})$ which is used to collect and aggregate the information from its cluster member sensor nodes and transfer that collected information to BS directly. Here, sensor nodes cannot send the information directly to BS rather they send it through $\mathrm{CH}$, so that's why it is called as hierarchical routing protocol. The whole process of LEACH is divided into 2 phases: set-up phase and steady-state phase. In set up phase, clusters are created and a $\mathrm{CH}$ is elected in that cluster by using the probability equation. Each sensor node generates a random number between 0 and 1 . If that generated number is less that threshold value then it will be $\mathrm{CH}$ for current round. The value of threshold is given by $\mathrm{T}(\mathrm{n})=\mathrm{P} /\{1-\mathrm{P}(\mathrm{r}$ mod $1 / \mathrm{P})\}$ where $\mathrm{P}$ is desired percentage of $\mathrm{CHs}, \mathrm{r}$ is the number of current round. And in steady state phase information transmission takes place between sensor nodes and $\mathrm{CH} \&$ $\mathrm{CH}$ and BS [24]. Its drawback is that it cannot be used for large area network because it uses one hop communication for transferring information from $\mathrm{CH}$ to BS.

In 2001, [15] Manjeshwar et al., introduces Threshold Sensitive Energy Efficient sensor Network (TEEN) protocol which is a hierarchical homogeneous clustering protocol. It is best suitable for time critical applications like intrusion detection, explosion detection etc., [24]. Here, $\mathrm{CH}$ does not send their information directly to the BS, instead it sends from lower level $\mathrm{CH}$ to one level above $\mathrm{CH}$ in hierarchy and so on until it reaches to the BS. After the process of clustering, the $\mathrm{CH}$ broadcast two threshold values to $\mathrm{SN}$ i.e., Hard threshold and Soft threshold. Hard threshold is used to reduce the amount of transmissions by allowing the SNs to send data only to those sensing element that are in their range of concern. However, Soft threshold is used to reduce the amount of transmission by overcoming all other transmissions which may have occurred when there is slight or no variation in the sense attribute. Here SNs will forward data in current round only, if, the existing value of sensed element is greater than hard threshold and if, the existing value of sensed element is different from previous sensed value by an amount equal to or greater than the soft threshold. It provides better improvement by reducing the number of redundant data transmission but at the cost of extra computational burden on SNs. Also it is not good for applications where the periodic information is generated.

In 2002, [16] LEACH was customized by Lindsey and Raghavendra and called it as Power Efficient Gathering in Sensor Information Systems (PEGASIS) protocol. The key thought in PEGASIS is to frame a chain among all sensor nodes so that each sensor nodes receives information from its closest neighbors; fuses its own information and pass it to the next sensor node. This process continues until information reaches to the destined BS [24]. This results in the reduction of average energy spent by each sensor node per round. However, this protocol is not suitable for large area networks due to excessive delay.

In 2004, Smaragdakis et al., introduces Stable Election Protocol (SEP) [17] which is an enhancement of LEACH in case of heterogeneity because LEACH protocol does not perform efficiently in case of sensor node heterogeneity. So, to overcome this, SEP was introduced which increases the network stability period and enhance the network lifetime. Here two types of heterogeneity are considered means two different types of sensor nodes are considered known as normal sensor nodes and advance sensor nodes. Advance nodes have more energy as compared to normal nodes and their quantity is also less due to its high cost. Here $\mathrm{CH}$ is selected on the basis of weighted election probability of sensor nodes [24]. So chances of advance 
sensor nodes to act as $\mathrm{CH}$ are more. Its drawback is that it is only used for 2 level of heterogeneity.

In 2006, Li et al., discussed Distributed Energy Efficient clustering (DEEC) [18] which is used for both 2-level and multi-level heterogeneity. Here $\mathrm{CH}$ is selected on the basis of probability equation which is the proportion of remaining energy of sensor node and average energy of network. So sensor nodes having more remaining energy have more chances to be $\mathrm{CH}$. It enhanced network lifetime and have more efficient data transmission technique as compared to SEP, LEACH and HEED [24]. Its drawback is that while designing multi-level heterogeneity large number of different energy level sensor nodes have to be used which may be not possible.

In 2007, Xiangning et al., introduces Multi-hop LEACH (MLEACH) [19] which tries to prevail over the scalability issue of LEACH along with multi-hop communication. But MLEACH fails when implemented in heterogeneous WSNs.

In 2009, Aderohunmu et al., discussed Enhanced Stable Election Protocol (ESEP) [20] which is enhancement of LEACH and SEP and provides 3-level heterogeneity. A new sensor node called as medium sensor node is introduced in the network along with normal and advance sensor node. Due to its distributed nature, it fails to attain possible number of $\mathrm{CHs}$ in its operation.

In 2012, Aslam et al., introduces a new routing protocols called Centralized Energy Efficient Clustering (CEEC) [21] which uses 3 level of heterogeneous network, where whole network area is partitioned into 3 equal regions and inside each region, sensor nodes with same energy level are deployed means different region have different sensor nodes energy. This method enhances the network lifetime and throughput.

In 2016, Khurana et al., introduces a new cluster based routing protocol called Multi-hop LEACH whose working operation is like that of LEACH [22]. But in Multi-hop LEACH, two major changes have been done i.e., multi-hoping concept is used for both communications that is in inter-cluster as well as intra-cluster. This improves the network lifetime as well as energy dissipation of whole network but it is implemented only in homogeneous WSNs.

In 2016, Jerbi et al., introduces the concept of Orphan LEACH (O-LEACH) [23] which was used to cover those sensor nodes which does not join any cluster during cluster formation phase which may be due to its far away location where $\mathrm{CH}$ cannot concentrate or these sensor nodes cannot reach to $\mathrm{CH}$. So this protocol increases the network connectivity and coverage but it is also implemented in homogeneous network.

In 2016, Cengiz et al., discussed a new algorithm called Low Energy Fixed Clustering Algorithm (LEFCA) [3,9] in which sensor nodes who became the member of particular $\mathrm{CH}$ during their set-up phase will remain inside that cluster throughout their network lifetime. This improves the network lifetime and decreases the network energy dissipation.

A state-of-art comparison of these existing cluster based routing protocols is presented in Table 1. Therefore, it can be concluded that WSNs is basically centered on technologies which are in light of homogeneous WSNs where all sensor nodes have same energy resources in previous works. However, heterogeneous WSNs are better known and a research indicates that it has prolonged the network lifetime as well as improves the reliability of network. Here sensor nodes have more intense and powerful microprocessor and memory as compared to normal homogeneous sensor nodes. They also have high bandwidth and can communicate to longer distance with BS.

The proposed EACBM routing protocol covers all these features like heterogeneity, scalability, multi-hoping, inter-cluster communication, connectivity, tackle unattended area and are well used for large area applications. 
Table 1. A State-Of-Art Comparison of Existing Cluster based Routing Protocols

\begin{tabular}{|c|c|c|c|c|c|c|}
\hline Features & $\begin{array}{c}\text { LEACH, } \\
\text { APTEEN, } \\
\text { PEGASIS }\end{array}$ & $\begin{array}{c}\text { MLEACH, } \\
\text { Multi-hop } \\
\text { LEACH }\end{array}$ & $\begin{array}{c}\text { SEP, } \\
\text { DEEC, } \\
\text { ESEP }\end{array}$ & CEEC & O-LEACH & LEFCA \\
\hline Heterogeneous & No & No & Yes & Yes & No & No \\
\hline Scalable & No & No & Yes & No & No & No \\
\hline $\begin{array}{c}\text { Tackles } \\
\text { unattended area }\end{array}$ & No & Yes & No & No & Yes & No \\
\hline Multi-hoping & No & Yes & No & No & No & No \\
\hline $\begin{array}{c}\text { Inter-cluster } \\
\text { Communication }\end{array}$ & No & Yes & No & No & No & No \\
\hline $\begin{array}{c}\text { Connectivity/ } \\
\text { Coverage }\end{array}$ & No & No & No & No & Yes & No \\
\hline $\begin{array}{c}\text { Applicable for } \\
\text { large area } \\
\text { network }\end{array}$ & No & No & No & No & No & No \\
\hline
\end{tabular}

\section{Proposed Approach}

In this section, authors have proposed a new cluster based heterogeneous routing protocol EACBM, which aims is to provide enhanced lifetime, scalability and energy efficiency of WSNs. It uses the concept of

- Clustering by grouping the sensor nodes into clusters and then elects a $\mathrm{CH}$ on the basis of new probabilistic equation which is responsible for aggregating and transmitting the sensed information to BS.

- Connectivity of far away sensor nodes with $\mathrm{CH}$ is also the major concern, so sub-clustering has been used to overcome this because it may be possible that those sensor nodes have some information which is necessary for particular application to make a decision.

- Multi-hopping is used for transmitting information if $\mathrm{CH}$ lies at far distance from BS.

The pseudo code for the selection of $\mathrm{CH}$ and multi-hop communication among $\mathrm{CHs}$ and $\mathrm{BS}$ in EACBM is: 


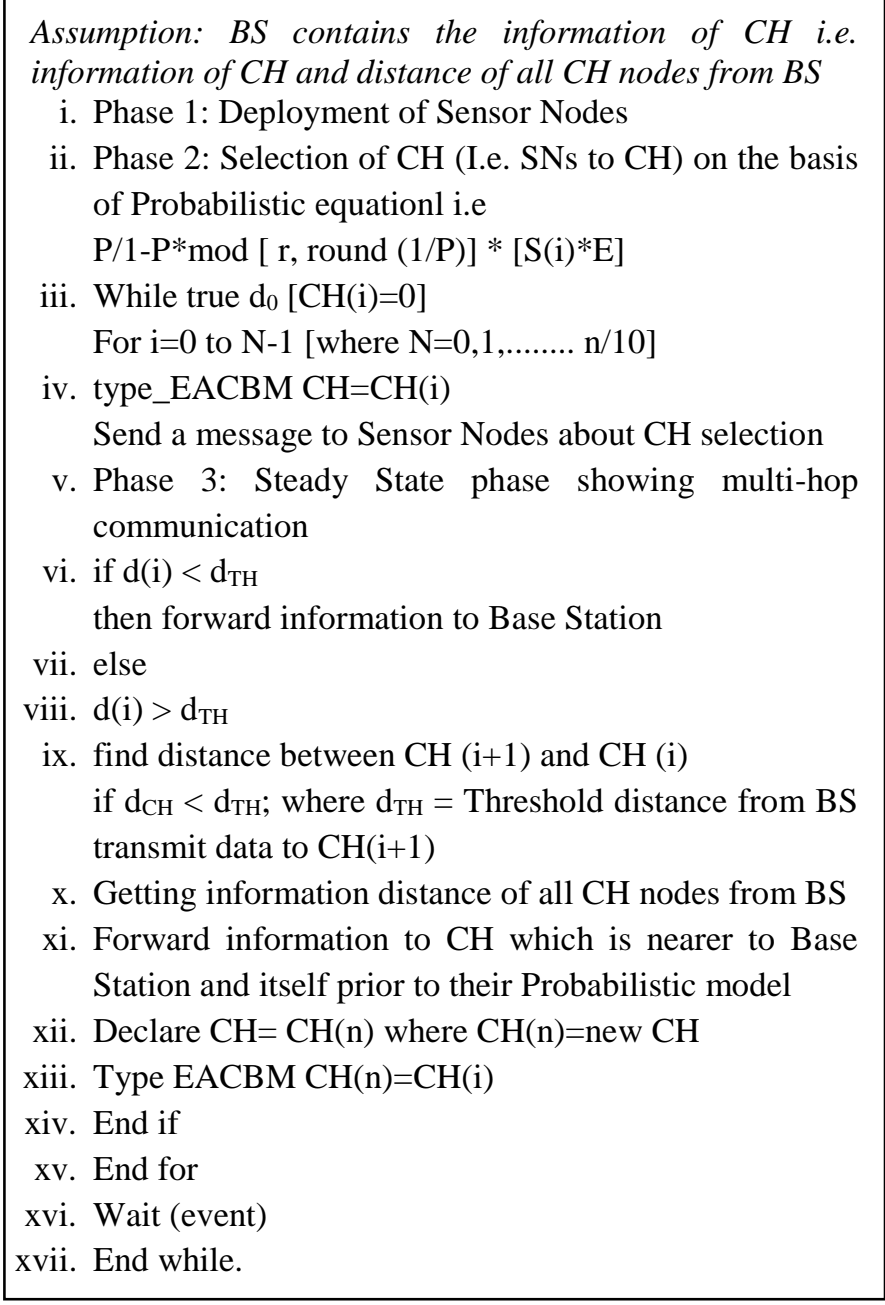

The working of EACBM heterogeneous cluster based routing protocol starts with the $\mathrm{CH}$ determination process performed by the BS. After the determination of $\mathrm{CHs}$, the set-up phase of EACBM starts in which clusters are formed and then steady state phase in which data transmission takes place between $\mathrm{CH}$ and BS. At the end, the $\mathrm{CH}$ reselection phase starts if required. These phases of EACBM protocol are explained below in details.

\subsection{Set-Up phase}

After the random deployment of sensor nodes in the entire field, this routing protocol initiates its set-up phase. EACBM protocol considers 3 types of heterogeneous sensor nodes (i.e., Normal, Intermediate and Advance SNs) having different types of energy level. Advanced SNs of fraction ' $m$ ' have $\alpha$ times more energy than normal SNs, i.e., $\mathrm{E}_{0}(1+\alpha)$. Whereas intermediate $\mathrm{SNs}$ of fraction ' $\mathrm{x}$ ' have $\beta$ times more energy than normal $\mathrm{SNs}$, i.e., $\mathrm{E}_{0}(1+\beta)$. As ' $\mathrm{N}$ ' is the total number of SNs in the network, then N.x.m, N.x. $(1-\mathrm{m}), \mathrm{N}(1-\mathrm{x})$ are number of advance, intermediate and normal SNs in the network respectively. With the addition of different energy level of SNs, the new epoch of network will be

$$
\frac{1}{P_{o p t}} \cdot(1+\alpha \cdot \mathrm{m}+\mathrm{x} \cdot \beta) .
$$

The initial energy of advance, intermediate and normal SNs are:

$$
\mathrm{E}_{\mathrm{adv}}=\mathrm{N} \cdot \mathrm{m} \cdot \mathrm{x} \cdot \mathrm{E}_{0} \cdot(1+\alpha)
$$




$$
\begin{aligned}
& E_{\text {int }}=N \cdot x \cdot(1-m) \cdot E_{0} \cdot(1+\beta) \\
& E_{n r m}=N \cdot(1-x) \cdot E_{0}
\end{aligned}
$$

So, the total initial energy of 3 level heterogeneous WSNs is given by:

$$
E_{\text {total }}=E_{a d v}+E_{\text {int }}+E_{n r m}
$$

The set-up phase stages are shown in Figure 1.

\begin{tabular}{|l|l|l|l|l|l|l|}
\hline CH selection & $\begin{array}{l}\text { Broadcast Adv } \\
\text { message }\end{array}$ & $\begin{array}{l}\text { Join request } \\
\text { messages }\end{array}$ & $\begin{array}{l}\text { TDMA schedule } \\
\text { messages }\end{array}$ & $\begin{array}{l}\text { Broadcast Adv } \\
\text { message }\end{array}$ & $\begin{array}{l}\text { Join request } \\
\text { messages }\end{array}$ & $\begin{array}{l}\text { TDMA schedule } \\
\text { messages }\end{array}$ \\
\hline
\end{tabular}

Figure 1. Set-up Phase of EACBM Routing Protocol

\subsubsection{CH Selection Phase:}

For selection of $\mathrm{CH}$, each sensor node produces an irregular number which lies between 0 and 1 and if that arbitrarily produced number is less than EACBM threshold value then only at that point that particular sensor node will be chosen as $\mathrm{CH}$ for that specific round. The value of new threshold value of EACBM is given by:

$$
\mathrm{T}\left(\mathrm{n}_{\mathrm{Z}}\right)= \begin{cases}\frac{\mathrm{P}_{\mathrm{Z}}}{1-\mathrm{P}_{\mathrm{Z}}\left(\mathrm{r} * \bmod \left(1 / \mathrm{P}_{\mathrm{Z}}\right)\right)} *[\mathrm{~S}(\mathrm{i}) . \mathrm{E}] & \text { if } \mathrm{n} \in \mathrm{G}^{\mathrm{Z}} \\ 0 & \text { otherwise }\end{cases}
$$

Where $\mathrm{P}_{\mathrm{Z}}$ is the probability of preferred number of SNs to be CHs, $\mathrm{Z}$ can be nrm, int, adv for Normal SNs, Intermediate SNs or Advance SNs individually, $r$ implies the tally of current round, [S(i).E] is existing energy of SN and it recommends that high energy SN ought be $\mathrm{CH}$ as compared to low energy SNs, G is the arrangement of SNs that had not been chosen as $\mathrm{CH}$ in the last $\mathrm{P}$ rounds.

The probability equation of every one of three sorts of sensor nodes should be different and is as per the following $\mathrm{P}_{\mathrm{nrm}}, \mathrm{P}_{\mathrm{int}}$ and $\mathrm{P}_{\mathrm{adv}}$ for Normal, Intermediate and Advance SNs individually:

$$
\begin{gathered}
\mathrm{P}_{\mathrm{nrm}}=\frac{\mathrm{P}_{\mathrm{opt}}}{1+\alpha \cdot \mathrm{m}+\mathrm{x} \cdot \beta} \\
\mathrm{P}_{\mathrm{int}}=\frac{\mathrm{P}_{\mathrm{opt}}(1+\beta)}{1+\alpha \cdot \mathrm{m}+\mathrm{x} \cdot \beta} \\
\mathrm{P}_{\mathrm{adv}}=\frac{\mathrm{P}_{\mathrm{opt}}(1+\alpha)}{1+\alpha \cdot \mathrm{m}+\mathrm{x} \cdot \beta}
\end{gathered}
$$

Where, $\mathrm{P}_{\text {opt }}$ is the optimal election probability of $\mathrm{SN}$ to be $\mathrm{CH}$.

\subsubsection{Cluster Formation Phase:}

After the selection of $\mathrm{CH}$, it notifies its status of being $\mathrm{CH}$ for current round to all other remaining sensor nodes in the network. For this, each $\mathrm{CH}$ generates an advertisement message (Adv) including $\mathrm{CH}-\mathrm{Id}$ and Adv Header using non-persistent Carrier Sense Multiple Access (CSMA) MAC protocol. Then each non-CH sensor nodes choose to which cluster they want to belong based on the $\mathrm{CH}$ Adv received signal strength (RSS). As sensor nodes choose their cluster, they must tell their related $\mathrm{CHs}$ about their selection. Every sensor node of clusters must send a join request message (join-REQ) to its selected $\mathrm{CH}$ using CSMA MAC protocol. This requested message must contains member sensor node ID, associated $\mathrm{CH}-\mathrm{Id}$, and a 
header. After the formation of clusters, every $\mathrm{CH}$ transmits a TDMA schedule inside its own cluster for the transmission of data by each sensor node. Inside this TDMA schedule, various time slots are allocated to each cluster member sensor nodes for transmitting its data. Also one extra slot is assigned for each $\mathrm{CH}$ to transmit its aggregated data to BS. The TDMA frame length depends upon the number of sensor nodes present inside each cluster +1 frame for $\mathrm{CH}$ of each cluster.

\subsubsection{Sub-Cluster Formation Phase:}

However, it may be the case that, in every round, there might be some sensor nodes who may not join any cluster because of its far away location and where $\mathrm{CH}$ range cannot be reached. So, to cover those sensor nodes a sub-cluster is created because may be those far away sensor nodes provides some pertinent information which have some extraordinary significance in some particular application. A sub$\mathrm{CH}$ is created in this sub-cluster which lies at halfway between cluster member sensor nodes and far away sub-clusters sensor nodes. The sub-cluster formation phase is same as that of cluster formation phase.

\subsection{Steady State Phase:}

The main goal of steady state phase is to forward the sensed information from sensor nodes to $\mathrm{CH}$ and to transfer the gathered and aggregated information from $\mathrm{CH}$ to the BS. This phase is little bit longer than setup phase. Also it has to decide whether a $\mathrm{CH}$ has to be changed or not for next round (Figure 2).

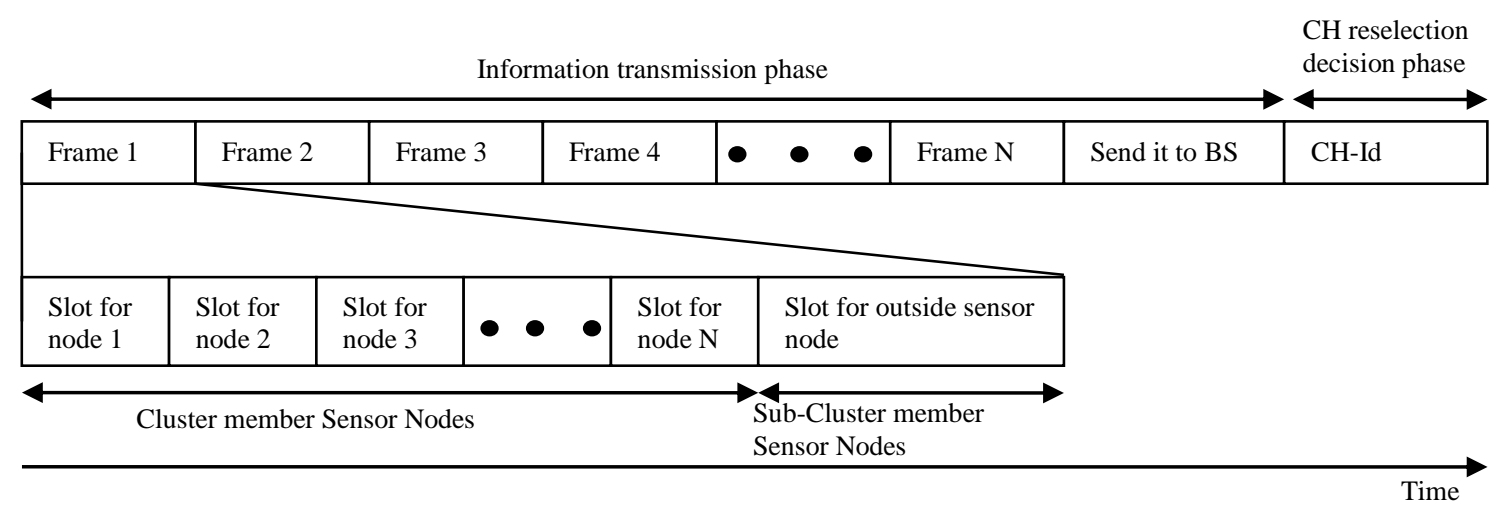

Figure 2. Steady State Phase of EACBM Routing Protocol

\subsubsection{Information Transmission Phase:}

In this phase, sensor nodes have to transmit their sensed information to the $\mathrm{CH}$ in the assigned TDMA frame. This process occurs in both cluster as well as subcluster. The information collected by sub- $\mathrm{CH}$ in sub-cluster must aggregated and transfer to the main $\mathrm{CH}$ of cluster. After the collection of information at $\mathrm{CH}$ from cluster member sensor nodes and as well as from sub- $\mathrm{CH}$, the $\mathrm{CH}$ has to aggregate all the collected information and forward it to the BS.

But before sending the aggregated information to the BS, CHs needs to verify whether BS is located near or it is far away. For this if the distance (di) between $\mathrm{CH}$ and $\mathrm{BS}$ is less than threshold distance $\left(\mathrm{d}_{\mathrm{TH}}\right)$, then it transmits the information directly to $\mathrm{BS}$ but if not then $\mathrm{CH}$ chooses the next $\mathrm{CH}$ which lies nearer to it and this process continues until aggregated information reaches the BS. The transmission of information in one cluster may cause affect in communication in the nearby cluster. So to reduce this interference inside clusters, a special CSMA 
spreading code has been sent. Every sensor node uses this spreading code during transmission of information so that no inter-cluster interference occurs.

\subsubsection{Decision for $\mathrm{CH}$ Reselection Phase:}

At the point when information transmission phase for a single round is finished, $\mathrm{CH}$ has to choose whether it will keep on acting as a $\mathrm{CH}$ for the following round or to pick another $\mathrm{CH}$. This choice of being $\mathrm{CH}$ is made on the basis of $\mathrm{CH}$ sesidual energy. If it is high as compared to other sensor nodes then it will continue to act as $\mathrm{CH}$ for the following round and must notify its cluster member sensor nodes with its $\mathrm{CH}$-Id otherwise new $\mathrm{CH}$ has to be selected and it notify its new $\mathrm{CH}$-Id to cluster member sensor nodes. This whole process of set-up phase and steady state phase proceeds until all sensor nodes are dead.

\section{Simulation and Performance Analysis of EACBM}

In this section, the performance of proposed EACBM routing protocol is compared with LEACH, SEP, CEEC and LEFCA cluster based routing protocols using MATLAB simulator. Network Lifetime, Stability Period, Numbers of CHs, Throughput, Numbers of dead nodes per round are the parameters selected for its evaluation.

The radio energy model is selected for heterogeneous WSNs to run the simulation. Transmitting sensor nodes consumes its energy for running the radio electronics and power amplifier whereas the receiving sensor nodes consumes its energy for running radio electronics [8]. Free space model and multi-path model are used for transmitting and receiving of information from $\mathrm{CH}$ to BS. The energy consumed by free space model is proportional to $\mathrm{d}^{2}$ (if $\mathrm{d} \leq \mathrm{d}_{0}$ ) and by multipath model is proportional to $d^{4}$ (if $d>d_{0}$ ) because the transmitting signal follows different paths to reach BS over the distance' $d$ ' is.

$$
E_{T X}(K, d)= \begin{cases}K \cdot E_{\text {elec }}+K \cdot \varepsilon_{f s} \cdot d^{2} \text { if } d \leq d_{0} \\ K \cdot E_{\text {elec }}+K \cdot \varepsilon_{m p} \cdot d^{4} \text { if } d>d_{0}\end{cases}
$$

$E_{\text {elec }}$ is the energy required per bit to active the transmitter or the receiver circuit, $\varepsilon_{\mathrm{fs}}$ and $\varepsilon_{\mathrm{mp}}$ are energy required by free space or multipath model respectively which depends upon the distance to the receiver (BS).

By equating both equations of $\mathrm{E}_{\mathrm{TX}}(\mathrm{K}, \mathrm{d})$ at $\mathrm{d}=\mathrm{d}_{0}$

$$
\mathrm{d}_{0}=\sqrt{\frac{\varepsilon_{\mathrm{fs}}}{\varepsilon_{\mathrm{mp}}}}
$$

Here, $\mathrm{d}_{0}$ is threshold distance which specifies the propagation transmission distance from direct path to multi-path model. If transmission distance is lesser than threshold distance $\left(\mathrm{d}_{0}\right)$ then the free space model are used otherwise, multipath model are used.

The amount of energy consumed for receiving K-bit of message is calculated by:

$$
\mathrm{E}_{\mathrm{RX}}(\mathrm{K})=\mathrm{K} \text {. } \mathrm{E}_{\text {elec }}
$$

Table 2 listed the simulation parameters and its values adopted [24,25]. 
Table 2. Parameter Settings

\begin{tabular}{|c|c|c|}
\hline Parameter & Meaning & Values \\
\hline Eelec & Electronic energy & $50 \mathrm{~nJ} / \mathrm{bit}$ \\
\hline EDA & Data aggregation energy & $\begin{array}{l}5 \mathrm{~nJ} / \mathrm{bit} / \\
\text { message }\end{array}$ \\
\hline $\mathbf{E}_{0}$ & Initial energy & $0.5 \mathrm{~J}$ \\
\hline $\mathbf{k}$ & Size of the message & 4000 bits \\
\hline $\mathbf{P}_{\text {opt }}$ & Probability & 0.1 \\
\hline$\varepsilon_{\text {fs }}$ & Friss free space & $10 \mathrm{pJ} / \mathrm{bit} / \mathrm{m}^{2}$ \\
\hline$\varepsilon_{\mathrm{mp}}$ & Friss multi-path & $\begin{array}{c}0.0013 \mathrm{pJ} / \mathrm{bit} / \\
\mathrm{m}^{4}\end{array}$ \\
\hline$\alpha$ & Energy level for Advance nodes & 3 \\
\hline $\boldsymbol{\beta}$ & $\begin{array}{c}\text { Energy level for Intermediate } \\
\text { nodes }\end{array}$ & 1.5 \\
\hline $\mathbf{m}, \mathbf{x}$ & $\begin{array}{c}\% \text { of nodes to be Advance and } \\
\text { Intermediate }\end{array}$ & $0.2,0.3$ \\
\hline $\mathbf{r}$ & Rounds & 5000 \\
\hline
\end{tabular}

Two different network scenarios have been considered for performance evaluation. In first scenario, 100 sensor nodes are randomly deployed in an area of $100 \mathrm{~m}$ by $100 \mathrm{~m}$ network area whereas in second scenario, 300 sensor nodes are randomly deployed in $500 \mathrm{~m}$ by $500 \mathrm{~m}$ network area.

\subsection{Performance Evaluation of EACBM with other Routing Protocols (LEACH, SEP, CEEC and LEFCA) with 100 Sensor Nodes Deployed in an Area of $100 \mathrm{~m}$ by 100m:}

Figure 3 shows comparison of the network lifetime/number of alive sensor nodes per round for all routing protocols. The performance of LEACH is far behind other routing protocols because it treats all sensor nodes equally without any energy discrimination. Whereas, SEP performs better than LEACH in terms of lifetime and stability because it deals with intial energy discrimation of all sensor nodes. Due to intelligent selection of $\mathrm{CH}$ in LEFCA makes it perform better than LEACH and SEP. While centralized cluster formation and planned sensor nodes deployment males CEEC performs better than LEACH, SEP and LEFCA. But, EACBM is far better compared to LEACH, SEP, CEEC and LEFCA in terms of network lifetime because of its energy heterogeneity awareness and the concept of multi-hoping that is used. The stability period of EACBM is almost $18 \%, 19 \%$ and $52 \%$ better than LEFCA, SEP and LEACH respectively and the network lifetime of EACBM is about $14 \%, 51 \%, 30 \%$ and $32 \%$ better than CEEC, LEFCA, SEP and LEACH respectively in this scenario. 


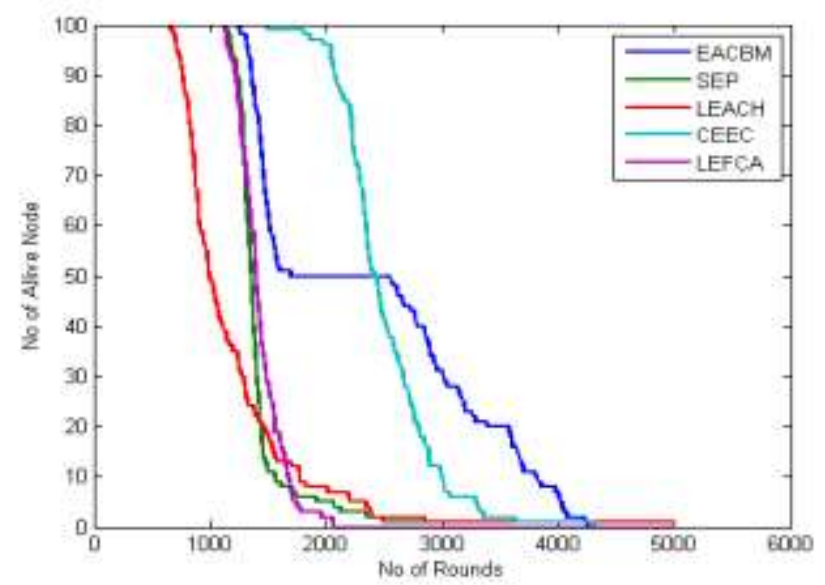

Figure 3. The Network Lifetime or Alive Nodes for $100 \mathrm{SNs}$ in $100 \mathrm{~m}$ by $100 \mathrm{~m}$

Figure 4 shows the number of $\mathrm{CHs}$ generated per rounds for all routing protocols. EACBM outperforms all other routing protocols (LEACH, SEP, LEFCA and CEEC) as it provides optimal number of $\mathrm{CHs}$ for every round. In some particular rounds; EACBM, CEEC, LEFCA, SEP, LEACH provides upto 23, 35, 55, 12 and 13 respectively number of $\mathrm{CHs}$.

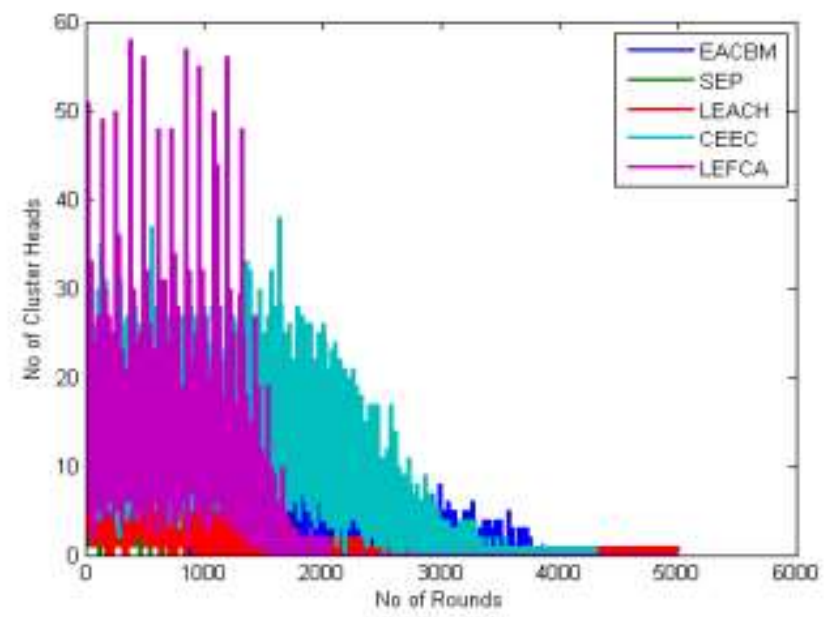

Figure 4. Number of CHs for $100 \mathrm{SNs}$ in $100 \mathrm{~m}$ by $100 \mathrm{~m}$

More number of $\mathrm{CHs}$ formed, more energy of network consumed. Due to distributed nature of $\mathrm{CH}$ selection in them, they perform poorly and does not provide optimal number of $\mathrm{CHs}$ that are required for efficient working of any protocol. CEEC performs better than LEACH, SEP and LEFCA because it provides optimal number of $\mathrm{CHs}$ for each round upto some extent. Due to uneven generation of $\mathrm{CHs}$ per round, it shows negative impact on the handling of network traffic which then results in the loss of packets due to insufficient TDMA slots.

In order to evaluate the features of any routing protocol, the successful data transmission of packets to BS is a crucial parameter. So, if BS is receiving high amount of data then that routing protocol is working properly. Figure 5 shows that EACBM outperforms other routing protocols in case of packets transmitted to BS. 


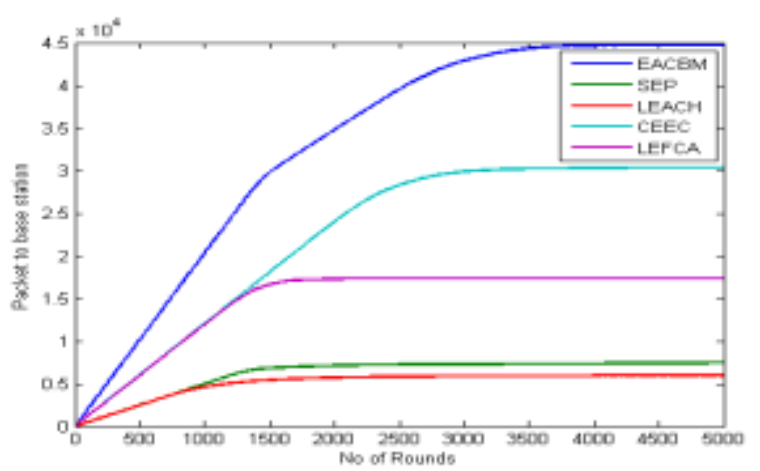

Figure 5. Packets transmitted to BS for $100 \mathrm{SNs}$ in $100 \mathrm{~m}$ by $100 \mathrm{~m}$.

Figure 6 illustrates the number of dead sensor nodes as the number of rounds increases. The increment of dead sensor nodes means higher the instability period of network. EACBM performs well and less number of sensor nodes is dead as compared to SEP, LEACH, CEEC and LEFCA with the passage of network operation. In EACBM, last sensor node dies after 4200 rounds whereas in SEP, LEACH, CEEC and LEFCA, approximately all SNs are dead much before that. So, it means the instability period of EACBM starts at later stage as compared to other routing protocols and it avoids the degradation of network performance as the network operation increases.

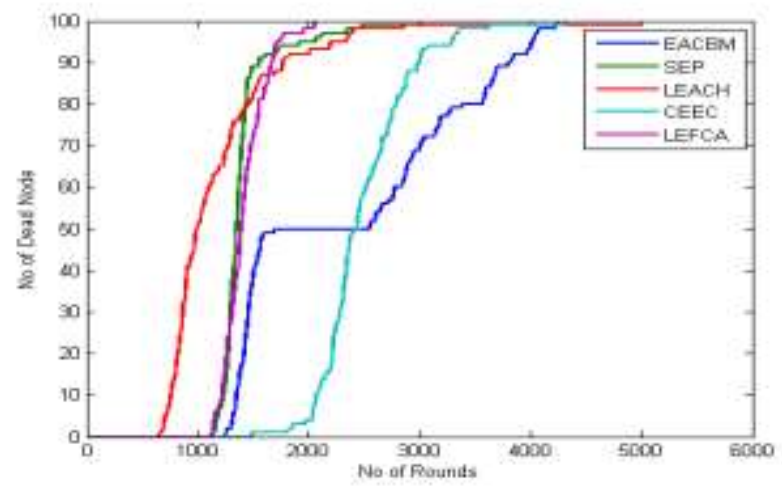

Figure 6. Number of Dead SNs for 100 SNs in $100 \mathrm{~m}$ by $100 \mathrm{~m}$

Table 3 shows the statistical value comparison between EACBM, CEEC, LEFCA, SEP and LEACH routing protocol under the first scenario, which is from the simulation results.

Table 3. Performance Comparison between EACBM, CEEC, LEFCA, SEP and LEACH

\begin{tabular}{|c|c|c|c|}
\hline Protocols & $\begin{array}{c}\text { Stability period } \\
\text { round }\end{array}$ & $\begin{array}{c}\text { In-stability period } \\
\text { round }\end{array}$ & Network Lifetime round \\
\hline EACBM & $1-1389$ & $1390-4248$ & $1-4248$ \\
\hline CEEC & $1-1492$ & $1493-3627$ & $1-3627$ \\
\hline LEFCA & $1-1129$ & $1130-2059$ & $1-2059$ \\
\hline SEP & $1-1121$ & $1122-2948$ & $1-2947$ \\
\hline LEACH & $1-654$ & $655-2855$ & $1-2855$ \\
\hline
\end{tabular}


4.2. Performance Evaluation of EACBM with other Routing Protocols (LEACH, SEP, CEEC and LEFCA) with 300 Sensor Nodes Deployed in an Area of 500m by 500m:

Simulation results specify that EACBM performs much better and shows considerable energy enhancement in terms of stability and network lifetime as compared to other routing protocols (LEACH, SEP, CEEC and LEFCA). Figure 7 indicates the network lifetime in this scenario. EACBM outperforms all other routing protocols due to better network management and provides improved stability period. All these are due to the use of mult-hoping concept and energy heterogeneity awareness of sensor nodes. The stability period of EACBM is approximately $62 \%$, $78 \%, 79 \%$ and $99 \%$ better than CEEC, LEFCA, SEP and LEACH respectively and the network lifetime of EACBM is about $16 \%, 51 \%, 53 \%$ and $56 \%$ better than CEEC, LEFCA, SEP and LEACH respectively in this scenario.

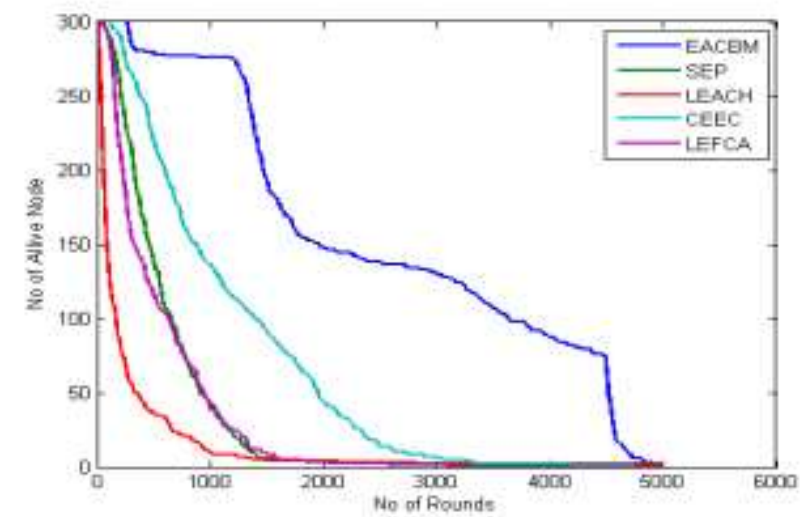

Figure 7. The Network Lifetime or Alive Nodes for 300 SNs in $500 \mathrm{~m}$ by $500 \mathrm{~m}$

The number of $\mathrm{CHs}$ generated per round becomes more uneven due to increase in the network area because with increase in network area, makes the intial phase more challenging and complex. Figure 8 shows the fluctuation in the number of $\mathrm{CH}$ per round in LEACH, SEP, CEEC and LEFCA has increased and reaches up to 150 as compared to previous scenario which shows up to 55. Whereas, EACBM provides optimal number of $\mathrm{CHs}$ for more number of rounds and such inefficiency hardly appears.

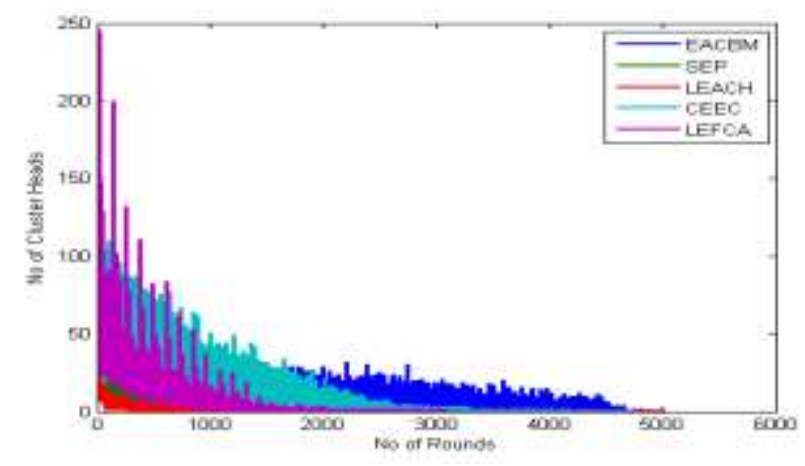

Figure 8. Number of CHs for $300 \mathrm{SNs}$ in $500 \mathrm{~m}$ by $500 \mathrm{~m}$

Figure 9 indicates the number of packets transmitted to BS and in that EACBM routing protocol outperforms other routing protocols. This is due to the uneven generation of $\mathrm{CHs}$ per round. 


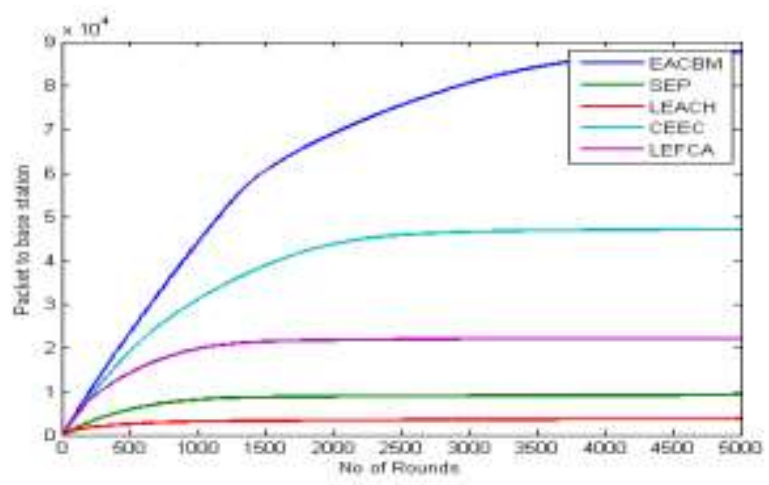

Figure 9. Packets Transmitted to BS for 300 SNs in $500 \mathrm{~m}$ by $500 \mathrm{~m}$

Figure 10 shows that the number of dead sensor nodes/ instability period of EACBM is very less as compared to other routing protocols.

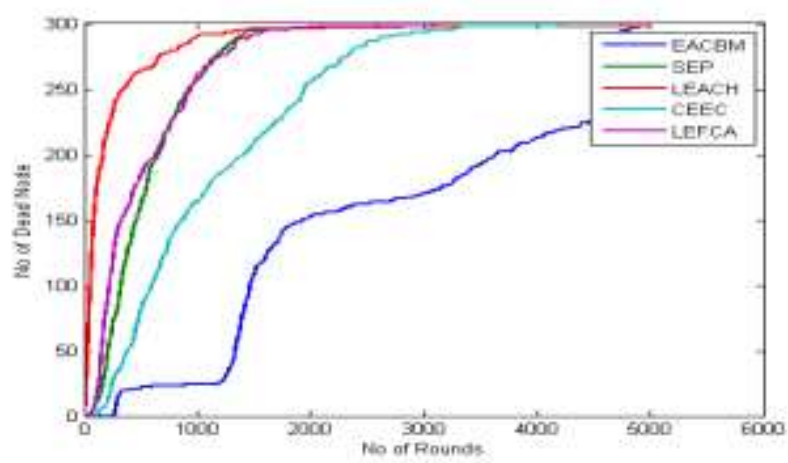

Figure 10. Number of Dead SNs for $300 \mathrm{SNs}$ in $500 \mathrm{~m}$ by $500 \mathrm{~m}$.

Here, approximately 150 sensor nodes are dead at around 2000 rounds in EACBM whereas in LEACH, SEP and LEFCA about $100 \%$ nodes are dead and in CEEC 250 sensor nodes are dead. Last sensor node in EACBM, dies after 4950 rounds. So EACBM, sends data to BS for more number of rounds.

Table 4 shows the statistical value comparison between EACBM, CEEC, LEFCA, SEP and LEACH routing protocol under the second scenario, which is from the simulation results.

Table 4. Performance Comparison between EACBM, CEEC, LEFCA, SEP and LEACH

\begin{tabular}{|c|c|c|c|}
\hline Protocols & $\begin{array}{c}\text { Stability period } \\
\text { round }\end{array}$ & $\begin{array}{c}\text { In-stability period } \\
\text { round }\end{array}$ & $\begin{array}{c}\text { Network Lifetime } \\
\text { round }\end{array}$ \\
\hline EACBM & $1-267$ & $268-4998$ & $1-4998$ \\
\hline CEEC & $1-99$ & $100-4199$ & $1-4199$ \\
\hline LEFCA & $1-58$ & $59-2409$ & $1-2409$ \\
\hline SEP & $1-54$ & $55-2348$ & $1-2348$ \\
\hline LEACH & $1-2$ & $3-2136$ & $1-2136$ \\
\hline
\end{tabular}




\section{Conclusion}

This paper proposes Energy Aware Cluster Based Multi-hop (EACBM) routing protocol using three level heterogeneous networks by combining the concept of clustering connectivity and multi-hop routing along with new probabilistic equation which results in the efficient utilization of sensor nodes energy. Only that sensor node will be $\mathrm{CH}$ whose energy or remaining energy is high as compared to others instead of selecting a random cluster member to be the next $\mathrm{CH}$. With this way, EACBM consumes the network energy in a conservative way and hence increases the lifetime of network. Results from simulation shows that, when EACBM is compared with traditional and novel cluster based routing protocols like LEACH, SEP, CEEC and LEFCA, significant improvement is achieved in case of network lifetime, stability, instability, throughput (packets transmitted to BS) and number of dead sensor nodes per rounds. Due to this, in future, we will see how our EACBM routing protocol works under the case of mobility.

\section{References}

[1] M. Arioua, Y. el Assari, I. Ez-zazi, and Ahmed El Oualkadi, "Multi-hop Cluster Based Routing Approach for Wireless Sensor Networks", Procedia Computer Science, vol. 83, (2016), pp. 584-591.

[2] A. Attea Bara'a and E. A. Khalil, "A new evolutionary based routing protocol for clustered heterogeneous wireless sensor networks", Applied Soft Computing, vol. 12, no. 7, (2012), pp. 19501957.

[3] K. Cengiz and T. Dag, "Improving Energy-Efficiency of WSNs through LEFCA", International Journal of Distributed Sensor Networks, vol. 12, no. 8, (2016), 8139401.

[4] I. F. Akyildiz, W. Su, Y. Sankarasubramaniam and E. Cayirci, "Wireless sensor networks: a survey", Computer networks, vol. 38, no. 4, (2002), pp. 393-422.

[5] M. Nasir Khan and M. Jamil, "Performance Improvement in Lifetime and Throughput of LEACH Protocol”, Indian Journal of Science and Technology, vol. 9, no. 21, (2016).

[6] J. N. Al-Karaki and A. E. Kamal, "Routing techniques in wireless sensor networks: a survey", IEEE wireless communications, vol. 11, no. 6, (2004), pp. 6-28.

[7] R. Kumari, A. Shantha Selva, A. Chithra and M. Balkis Devi, "Efficient 2-level energy heterogeneity clustering protocols for wireless sensor network", Indian Journal of Science and Technology, vol. 9, no. $8,(2016)$.

[8] K. Tae Kim and H. Yong Youn, "A stochastic and optimized energy efficient clustering protocol for wireless sensor networks", International Journal of Distributed Sensor Networks, vol. 10, no. 3, (2014), 828675 .

[9] K. Cengiz and T. Dag, "Multi-hop low energy fixed clustering algorithm (M-LEFCA) for WSNs", IEEE 3rd International Symposium on in Telecommunication Technologies (ISTT), IEEE, (2016), pp. 31-34.

[10] M. Sabet Maryam and H. Naji, "An energy efficient multi-level route-aware clustering algorithm for wireless sensor networks: A self-organized approach", Computers \& Electrical Engineering, vol. 56, (2016), pp. 399-417.

[11] N. Javaid, T. N. Qureshi, A. H. Khan, Adeel Iqbal, E. Akhtar and M. Ishfaq, "EDDEEC: Enhanced developed distributed energy-efficient clustering for heterogeneous wireless sensor networks", Procedia Computer Science, vol. 19, (2013), pp. 914-919.

[12] W. R. Heinzelman, A. Chandrakasan, H. Balakrishnan, "Energy efficient routing protocols for wireless microsensor networks", 33rd Hawaii International Conference on System Sciences, vol. 8, (2002), pp. 8020-8030.

[13] W. Jerbi, A. Guermazi and H. Trabelsi, "A novel clustering algorithm for coverage a large scale in WSN", arXiv preprint arXiv:1605.03079, (2016).

[14] W. Rabiner Heinzelman, A. Chandrakasan and H. Balakrishnan, "Energy-efficient communication protocol for wireless microsensor networks", Proceedings of the 33rd annual Hawaii international conference on in System Sciences, IEEE, (2000), pp. 10.

[15] A. Manjeshwar and D. P. Agrawal, "TEEN: ARouting Protocol for Enhanced Efficiency in Wireless Sensor Networks", In ipdps, vol. 1, (2001), pp. 189.

[16] S. Lindsey and C. S. Raghavendra, "PEGASIS: Power-efficient gathering in sensor information systems", In Aerospace conference proceedings, IEEE, vol. 3, (2002), pp. 3-3.

[17] G. Smaragdakis, I. Matta and A. Bestavros, "SEP: A stable election protocol for clustered heterogeneous wireless sensor networks", Boston University Computer Science Department, (2004).

[18] L. Qing, Q. Zhu and M. Wang, "Design of a distributed energy-efficient clustering algorithm for heterogeneous wireless sensor networks", Computer communications, vol. 29, no. 12, (2006), pp. 22302237. 
[19] F. Xiangning and S. Yulin, "Improvement on LEACH protocol of wireless sensor network", In International Conference on Sensor Technologies and Applications, SensorComm, IEEE, (2007), pp. 260-264.

[20] F. A. Aderohunmu and J. D. Deng, "An enhanced stable election protocol (sep) for clustered heterogeneous wsn”, Department of Information Science, University of Otago, New Zealand, (2009).

[21] M. Aslam, T. Shah, N. Javaid, A. Rahim, Z. Rahman and Z. A. Khan, "CEEC: Centralized energy efficient clustering a new routing protocol for WSNs", In 9th Annual IEEE Communications Society Conference on Sensor, Mesh and Ad Hoc Communications and Networks (SECON), (2012), pp. 103105.

[22] S. Khurana and K. Rawat Rekha, "Energy Efficient of Inter Cluster Multihop Routing Protocol for Wireless Sensor Network", (2016).

[23] W. Jerbi, A. Guermazi and H. Trabelsi, "O-LEACH of routing protocol for wireless sensor networks", In 13th International Conference on Computer Graphics, Imaging and Visualization (CGiV), IEEE, (2016), pp. 399-404.

[24] A. S. Toor and A. K. Jain, "A survey of routing protocols in Wireless Sensor Networks: Hierarchical routing”, 2016 International Conference on Recent Advances and Innovations in Engineering (ICRAIE), Jaipur, India, (2016), pp. 1-6.

[25] T. Sharma, B. Kumar and G. Singh Tomar, "Performance Comparision of LEACH, SEP and DEEC Protocol in Wireless Sensor Network", In Proc. of the Intl. Conf. on Advances in Computer Science and Electronics Engineering, (2012).

\section{Authors}

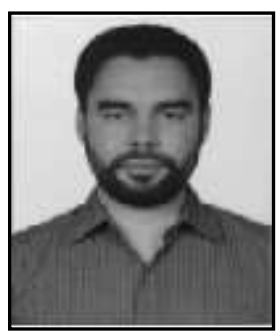

Amanjot Singh Toor was born in Hoshiarpur, Punjab, India on June 04th, 1987. He received B. Tech Degree in Electronics \& communication Engineering from SBBSIET, Jalandhar, Punjab, India in 2010 and M. Tech Degree in Electronics \& communication Engineering from GNE College, Ludhiana, Punjab, India in 2013. He is Research Scholar at Department of Instrumentation and Control Engineering, Dr. B. R. Ambedkar National Institute of Technology, Jalandhar, Punjab, India. He has published several papers in National \& International Conferences, International Journals on Wireless Sensor Network, Optical Communication. His research area of interest is Wireless Sensor Networks.

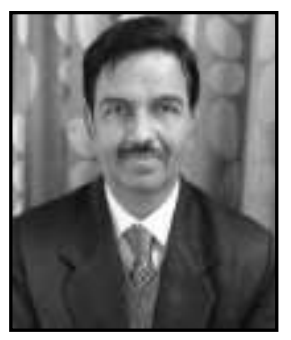

A. K. Jain received his B.E and M.E both from IIT, Roorkee, (erstwhile University of Roorkee, Roorkee) India in 1981 and 1987 respectively and received his Ph.D degree on Quality of Service in High Speed Networks from the Dr. B. R. Ambedkar National Institute of Technology, Jalandhar, India in 2009. He has published over sixty research papers in national and international journals/conferences. He is presently working as Professor in the Department of Instrumentation and Control Engineering, Dr. B. R. Ambedkar National Institute of Technology, Jalandhar, India. $\mathrm{He}$ is guiding Ph.D and M.Tech students in the area of Wireless Networks. Before joining N.I.T, Jalandhar, he has served at TIET Patiala, IET Lucknow, and NIT Hamirpur (Erstwhile REC Hamirpur) in various capacities. His research interests include quality of service in Wireless Networks, Wireless Sensor Network, Medium Access Protocols for mobile computing, and mesh networks. Dr. Jain is member of IEEE and ISTE India 